\title{
Congenital fistula between the left ventricle and coronary sinus: elucidation by colour Doppler flow
} mapping

\author{
J P GNANAPRAGASAM, A B HOUSTON, S LILLEY \\ From the Departments of Cardiology and Child Health, Royal Hospital for Sick Children, Glasgow
}

SUMMARY A fistula between the left ventricle and the coronary sinus was diagnosed by Dopple echocardiography and confirmed by cardiac catheterisation in a symptom free child who presenteథ with clinical signs of mitral regurgitation. A similar abnormality has been reported after repeated mitral valve replacement and after myocardial infarction but a congenital fistula of this type has no席 been described before.

Congenital abnormalities of the coronary sinus are uncommon. They include abnormal systemic or pulmonary venous drainage into the coronary sinus, fistulas between the coronary artery and coronary sinus, absence of the coronary sinus, atresia of the coronary sinus ostium, ${ }^{1}$ and coronary sinus aneurysms. ${ }^{23}$ The development of an iatrogenic fistula between the left ventricle and the coronary sinus is a recognised surgical complication after repeated prosthetic mitral valve replacement. ${ }^{45}$ An acquired fistula has also been reported as a complication of acute myocardial infarction. ${ }^{6}$ We report the case of a child with a congenital fistula between the left ventricle and the coronary sinus, a congenital abnormality that has not been described before.

\section{Case report}

A 20 month old girl was found to have an asymptomatic heart murmur on routine examination. Her previous history and family history were unremarkable. Physical examination showed a well nourished child with no cyanosis or clubbing and normal pulses. The apex beat was not displaced and there was no precordial thrill. Auscultation showed normal heart sounds with a grade $2 / 6$ long systolic murmur best heard over the apex and radiating to the axilla and back. There was no other murmur. A clinical diagnosis of mitral regurgitation was made.

Electrocardiography was normal. Cross sectional imaging showed normal mitral valve leaflets and papillary muscle attachments. Stand alone continuous wave Doppler examination showed a high

Requests for reprints to Dr J P Gnanapragasam, Royal Hospital for Sick Children, Yorkhill, Glasgow G3 8SJ. velocity systolic jet (velocity $4.2 \mathrm{~m} / \mathrm{s}$, equivalent to $\vec{a}$ pressure gradient of $70 \mathrm{~mm} \mathrm{Hg}$ ) in the region of the mitral valve, which was interpreted as being in keeping with the clinical diagnosis of mild mitras regurgitation.

Review when she was five years old showed that she was symptom free with no change in the physicado signs. Echocardiography was performed with a Vingmed CFM 700 system with duplex pulsed and continuous wave and colour Doppler flow mapping Colour Doppler clearly showed a high velocity jeø from the left ventricle through its posterior wall into\% an enlarged coronary sinus (fig 1). Doppler examinae tion at a high frequency of pulse repetition and with the sample volume placed over the jet seen on coloug mapping confirmed a systolic jet with a velocity of about $4 \mathrm{~m} / \mathrm{s}$ (pressure difference $64 \mathrm{~mm} \mathrm{Hg}$ ). Tho spectral record showed both a fixed obstructive signal and a dynamic one, which indicated increasing obstruction during systole.

Subsequent careful ultrasound examination showed that when the scanning plane was tilted to the right, the medial part of the coronary sinus could be shown to be dilated. Colour Doppler flow mapping showed a communication between the left ventricle and the coronary sinus. There was no evidence of mitral regurgitation or other abnormality.

Cardiac catheterisation was undertaken to confirm this unusual abnormality. Left ventricular angio? cardiography produced immediate opacification of the coronary sinus (fig 2a) before filling of the्E coronary arteries had occurred. The coronary arteries were normal. The cardiac veins draineg normally into an aneurysmally dilated coronary sinuef (fig 2b). The oxygen saturation in the peripheral pare of the coronary sinus was $38 \%$ and in the dilated medial part it was $95 \%$. This indicated left to right 

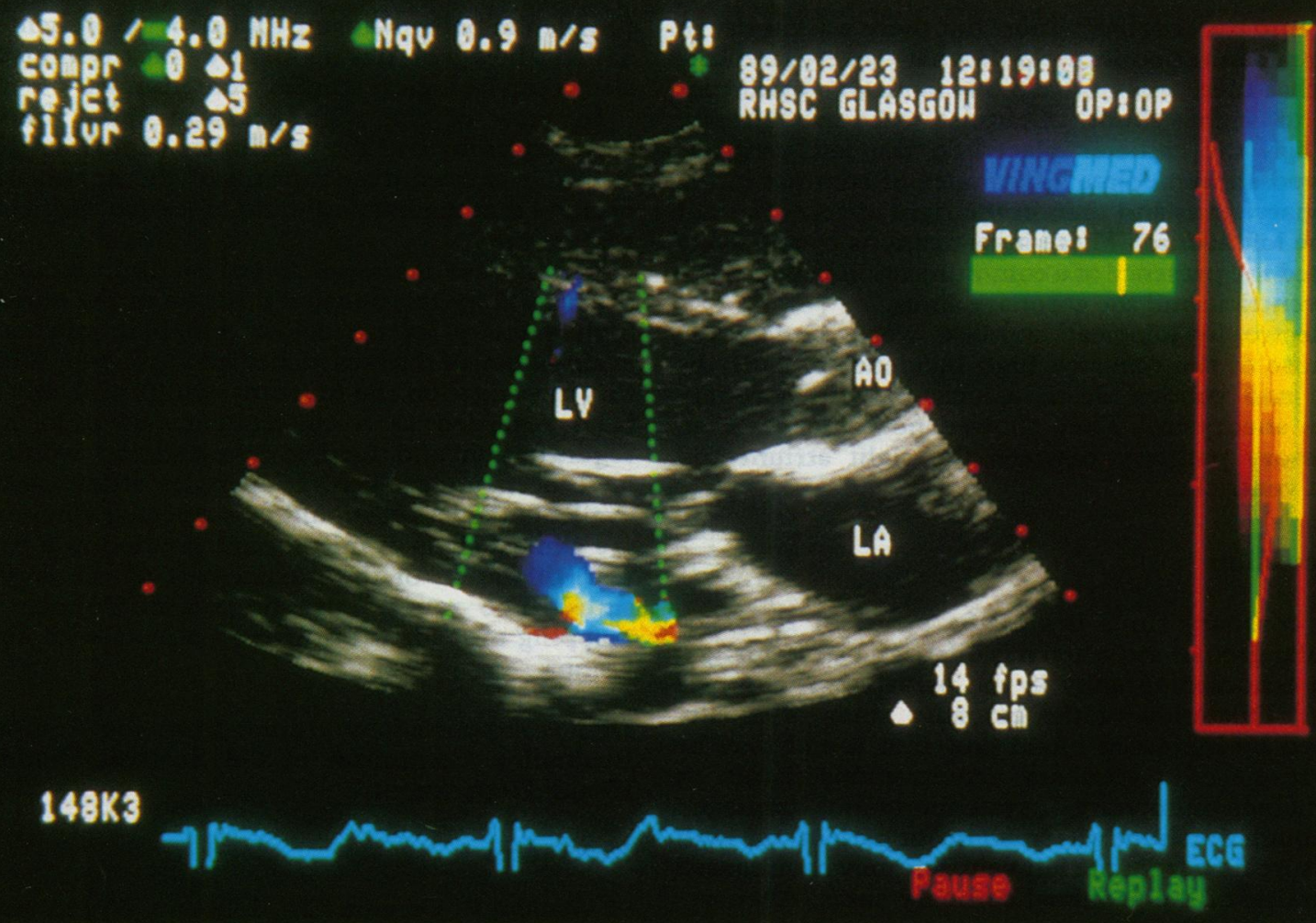

Fig 1 Parasternal long axis view with colour Doppler flow mapping showing high velocity flow from the base of the left ventricle into the coronary sinus, which is dilated. $L V$, left ventricle; $L A$, left atrium; Ao, aorta.

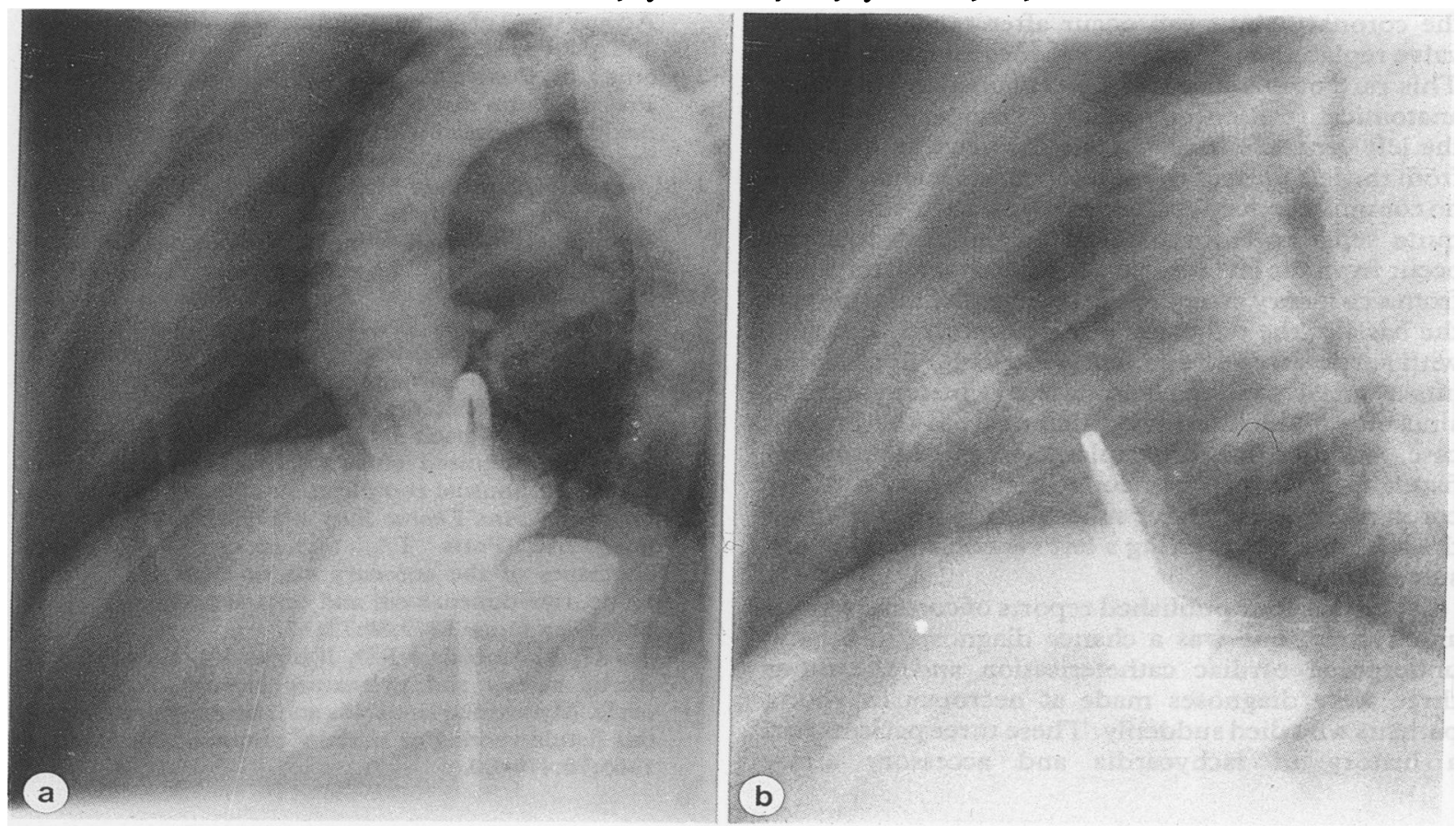

Fig 2 (a) Left ventricular angiocardiogram showing filling of the aneurysmal coronary sinus. (b) Angiogram after an injection of contrast into the coronary sinus. 
flow into the coronary sinus, but there was no rise in oxygen saturation in the pulmonary artery, indicating that there was no haemodynamically significant left to right shunt. Contrast echocardiography through the catheter placed in the left ventricle showed an immediate appearance of contrast in the coronary sinus and then in the right atrium, but not in the left atrium. These investigations conclusively proved the presence of a communication between the left ventricle and the coronary sinus.

Electrophysiological studies were undertaken because of the reported high incidence of arrhythmias and sudden death associated with coronary sinus aneurysm. ${ }^{3}$ They showed no evidence of an accessory pathway between the right atrium and right ventricle.

\section{Discussion}

The coronary sinus lies in the posterior atrioventricular groove and when it is dilated it can be imaged with echocardiography in the parasternal long axis view. ${ }^{7}$ Dilatation of the coronary sinus is usually caused by increased blood flow into it and may be the result of a coronary arteriovenous fistula or anomalous pulmonary or systemic venous drainage. ${ }^{1}$ Although the shunt was small in our patient, the high pressure flow from the left ventricle may have caused dilatation of the proximal coronary sinus. Aneurysmal dilatation of the coronary sinus may also occur as an isolated defect without a shunt into the sinus. ${ }^{23}$

An acquired fistula between the left ventricle and the coronary sinus can occur after repeated mitral valve replacement ${ }^{45}$ and after myocardial infarction. ${ }^{6}$ This rare occurrence can be explained by the close anatomical relation between the coronary sinus and the left ventricle. Because the coronary sinus arises from the left horn of the sinus venosus it should have no communication with the left ventricle, which has a quite separate embryological origin. A fistula can occur from the left ventricle to a coronary artery, and from a coronary artery to the coronary sinus; this was the basis of the only similar case reported, a patient with aortic valve atresia, left ventricular hypoplasia, intact atrial septum, and dilated intramyocardial sinusoids, ${ }^{8}$ which is very different from the present case. Another possible explanation for this unusual fistula is that a primary congenital aneurysm of the coronary sinus may have subsequently ruptured into the left ventricle creating a left ventricle to coronary sinus fistula.

There are four published reports of coronary sinus aneurysm ${ }^{23}$; one was a chance diagnosis in a baby undergoing cardiac catheterisation and the other three were diagnoses made at necropsy in young patients who died suddenly. These three patients had a history of tachycardia and accessory atrio- ventricular pathways were found in two of them. Our patient, however, has no history of arrhythmias and electrophysiological studies were normal.

In view of the high pressure shunt into theo coronary sinus and the possibility of further dilata-등 tion and extracardiac rupture of the aneurysm we decided that the fistula should be closed surgically at $\widehat{\nabla}$ a later date.

In this case the suspicion of an abnormality was first raised by the unusual position of the abnormal $\vec{\circ}$ high velocity jet on colour Doppler and carefulexamination of the area identified the fistula. Without $\vec{\omega}$ this investigation the murmur would probably have $\frac{\rho}{f}$ been regarded as a consequence of mild mitral? regurgitation and further investigation would not $N$ have been undertaken. If Doppler studies are noter available the lesion should be suspected where there $\dot{\circ}$ are clinical signs of mitral regurgitation in a patient ${ }^{\circ}$ with dilatation of the medial part of the coronarys sinus. The routine use of colour Doppler flow mapping may identify further patients with similar lesions.

JPG is supported by a grant from the Equipment Evaluation Committee of the Scottish Home and Health Department.

\section{References}

1 Mantini E, Grondin CM, Lillehei CW, Edwards JE. Congenital anomalies involving the coronary sinus. Circulation 1966;33:317-27.

2 Ho SY, Gupta I, Anderson RH, Lendon M, Kerr I. Aneurysm of the coronary sinus. Thorax $\overrightarrow{\bar{O}}$ 1983;38:686-9.

3 Gerlis LM, Davies MJ, Boyle R, Williams G, Scott H. Pre-excitation due to accessory sinoventricular connexions associated with coronary sinus aneurysms: areport of two cases. Br Heart J 1985;53:314-22.

4 Morritt GN, Jamieson MPG, Irving JB, Marquis RM, Walbaum PR. Development of left ventricular-? coronary sinus fistula following replacement of mitral valve prosthesis. J Thorac Cardiovasc Surg 1978; 76:381-4.

5 Miller DC, Schapira JN, Stinson EB, Shumway NE. Left ventricular-coronary sinus fistula following 응 repeated mitral valve replacements. $J$ Thorac Cardiovasc Surg 1978;76:43-5.

6 Mahfood SS, Watson DC, Di Sessa TG, Nunn SL, Ratts TE. Acquired left ventricular to coronary sinus $N$ fistula: an unusual complication of acute myocardial infarction. Ann Thorac Surg 1987;44:550-1.

7 Snider AR, Ports TA, Silverman NH. Venous anomalies of the coronary sinus: detection by $M-O$ mode, two-dimensional and contrast echocardiography. Circulation 1979;60:721-7.

8 Ragis G, Bloemendaal RD, Kanyah VI, Edwards JE. Ф Aortic atresia and premature closure of foramen? ovale. Myocardial sinusoids and coronary arterioven- $T$ ous fistula serving as outflow channel. Am Heart $J$ 1965;70:476-80. 